\title{
Estimation of Minimal Length Using Binding Energy of Deuteron
}

\author{
M. Moniruzzaman ${ }^{1 *}$, S. B. Faruque ${ }^{2}$ \\ ${ }^{1}$ Department of Physics, Mawlana Bhashani Science and Technology University, Santosh, Tangail- \\ 1902, Bangladesh \\ ${ }^{2}$ Department of Physics, Shahjalal University of Science and Technology, Sylhet, 3114, Bangladesh
}

Received 8 June 2017, accepted in final revised form 26 January 2018

\begin{abstract}
We find the shift of ground state energy of deuteron caused by generalized uncertainty principle (GUP) using Yukawa potential as the binding force between the proton and neutron. This leads to an upperbound on minimal length of about $10^{-18} \mathrm{~m}$ which is close to the values frequently found in literature.
\end{abstract}

Keywords: Generalized uncertainty principle; Minimal length; Yukawa potential; Ground state energy of deuteron.

(C) 2018 JSR Publications. ISSN: 2070-0237 (Print); 2070-0245 (Online). All rights reserved. doi: http://dx.doi.org/10.3329/jsr.v10i2.32829

J. Sci. Res. 10 (2), 99-103 (2018)

\section{Introduction}

A minimal observable length manifest theoretically through a non-zero minimal uncertainty $(\Delta \mathrm{x})_{\min }$ in position measurements [1-3] is nowadays a very interesting and important element of physics research. Many versions of quantum gravity [4], string theory [5] and non-commutative geometry [6] predict such a minimal observable length. Quantum mechanics gets considerable modification due to the incorporation of minimal length via generalized uncertainty principle (GUP), which is $\Delta x \Delta p \geq \frac{\hbar}{2}\left(1+\beta(\Delta p)^{2}\right)$, where $\beta$ is a small parameter, assumed to be positive [7]. This modified uncertainty relation gives rise to $(\Delta x)_{\min }=\hbar \sqrt{\beta}$ in one dimension, which is theorized to be around the Planck length, but can be much different since it is yet an unobserved. The most significant upperbound on $(\Delta x)_{\min }$ is $\approx 10^{-17} \mathrm{~m}$ which is found by comparing experimental uncertainty of electron energy in hydrogen atom with the theoretical shift of energy of the same caused by GUP [8,9]. Although, it is the Planck length $\left(\approx 10^{-35} \mathrm{~m}\right)$ where GUP is thought and theorized to be most operational, GUP can have observable consequences at length scales much larger than the Planck scale which is evident from the modified uncertainty

Corresponding author: 
relation (since it contains a UV/IR mixing) and the upperbound just quoted. We have previously presented an upperbound on minimal length from shift caused by GUP to deuteron quadrupole moment which is about $10^{-16} \mathrm{~m}$ [10]. In the present paper, we estimate the correction to the ground state energy of deuteron due to GUP and use that to estimate an upperbound on minimal length.

\section{The Influence of the Generalized Uncertainty Principle on the Ground State Energy of Deuteron and the Estimation of Minimal Length}

The ground state of deuteron is an admixture of ${ }^{3} S_{1}$ and ${ }^{3} D_{1}$ states. Since the exact form of the neutron-proton interaction potential by which deuteron is bound is not fully known, one usually cannot predict the binding energy of deuteron. The simplest approach, however, to find the wave function of deuteron is to use a square-well potential. Here, there are two free parameters: the depth and the range of the potential. One can at best find, using the known data, the range-depth relationship. The situation somewhat improves if one uses the Yukawa potential given by

$V(r)=-A \frac{e^{-\alpha r}}{r}$

where the parameter $A$ is dependent on both the depth and range of the interaction, and $\alpha$ is solely dependent on the range. The Schroedinger equation to be solved is of the form

$$
\left[\frac{\vec{p}^{2}}{2 \mu}+V(\vec{r})\right] \psi(\vec{r})=E \psi(\vec{r})
$$

which modifies, when generalized uncertainty principle is taken into account, to [8]

$$
\left[\frac{\vec{p}^{2}}{2 \mu}+\frac{\beta}{\mu} \vec{p}^{4}+V(\vec{r})\right] \psi(\vec{r})=E \psi(\vec{r})
$$

where $\mu$ is the reduced mass of deuteron and other symbols represent usual dynamical quantities.

We are interested to know how the energy eigenvalue $E$ in Eq.(3) differs from the $E$ in Eq.(2), due to the term $\frac{\beta}{\mu} \vec{p}^{4}$ which is the contribution of GUP to the Hamiltonian. To accomplish our task, we shall use an approximation of the potential (1) with only the most dominant terms in its expansion, i.e.

$V(r) \approx-\frac{A}{r}+A \alpha$

and we shall use the experimentally known value of the binding energy of deuteron which is $2.224 \pm 0.000009 \mathrm{MeV}$ [11]. Moreover, we shall use a precise value of $\alpha=\left(\frac{\hbar}{m_{\pi} c}\right)^{-1}=\left(1.43 \times 10^{-15} \mathrm{~m}\right)^{-1}, m_{\pi}$ is the mass of pion and $\frac{\hbar}{m_{\pi} c}$ is the Compton wavelength of pion and it is the most plausible range of nuclear interaction operational in deuteron in the leading order. By such a procedure, we reduce the set of free parameters in our model to only one, namely, $A$. We can solve Eq. (2) first with the potential given by the first term in Eq. (4); the problem then is like solving the hydrogen atom problem in non-relativistic quantum mechanics. Thereafter, we can find the contribution of the second term of Eq. (4) using simple perturbation technique. We, then, pose an algebraic equation to find the 
value of $A$ in the present model. Subsequently, we can find the contribution of the term $\frac{\beta}{\mu} \vec{p}^{4}$ of Eq. (3) and find the correction to the energy of deuteron due to GUP posed by Eq.(3). Throughout the perturbation calculation we can use hydrogen atom like wavefunctions as the unperturbed wavefunctions. The procedure outlined here is justified as long as we aim to find an order of magnitude shift of the binding energy of deuteron due to GUP.

Now, using the potential $-\frac{A}{r}$ in Eq.(2), and measuring distance in units of $\frac{\hbar^{2}}{\mu A}$ and $E$ in units of $\frac{\mu A^{2}}{2 \hbar^{2}}$, we have the transformed Eq.(2) as follows:

$$
\nabla^{2} \psi+\left(E+\frac{2}{r}\right) \psi=0
$$

whose solution is well known in quantum mechanics (see, for example, [12]), and the energy eigenvalue is given by

$E_{n}=-\frac{1}{n^{2}} \equiv-\frac{\mu A^{2}}{2 \hbar^{2}} \frac{1}{n^{2}}$

Moreover, the radius of the ground state orbit is given by $a_{0}=\frac{\hbar^{2}}{\mu A}$. We do not write the wavefunctions for they are well known and can be found in textbooks (for example, see [12]). The contribution of the term $A \alpha$ to energy is $A \alpha$. Hence, we can pose the following algebraic equation to find a particular value of $A$ :

$\frac{\mu A^{2}}{2 \hbar^{2}}-A \alpha=E_{0}$

Using the binding energy $E_{0}=2.224 \mathrm{MeV}, \frac{1}{\alpha}=1.43 \times 10^{-15} \mathrm{~m}$ and known value of $\mu$, we find from Eq. (7),

$A=1.89 \times 10^{-26} \mathrm{Jm}$

where positivity of $A$ was assumed in evaluation from Eq. (7). This value of $A$ corresponds to depth of the potential of about $82.5 \mathrm{MeV}$, which is very plausible and close to the values frequently found in literature. Now we can proceed to find the contribution of the term $\frac{\beta}{\mu} \vec{p}^{4}$ of Eq. (3) to the energy and equate that to the experimental uncertainty in $E_{0}$ which is, as quoted above, $\pm 0.000009 \mathrm{MeV}$. A straight forward calculation gives

$\left\langle\frac{\beta}{\mu} \vec{p}^{4}\right\rangle \equiv \Delta E_{0}=\beta \frac{\mu^{3} A^{4}}{\hbar^{4}}=6.12 \times 10^{-50} \beta$

The three dimensional expression for $(\Delta x)_{\min }=\hbar \sqrt{5 \beta}$. Using this in Eq. (9) and the experimental $\Delta E_{0}$ quoted just, we get an upperbound on minimal length, if we attribute the whole uncertainty in binding energy of deuteron to GUP, as $(\Delta x)_{\min } \leq 1.6 \times 10^{-18} \mathrm{~m}$

This upperbound on minimal length is within the span of values that are frequently found from other systems like hydrogen atom $\left((\Delta x)_{\min } \sim 10^{-17} \mathrm{~m}[8]\right)$, deuteron qudrupole moment $\left((\Delta x)_{\min } \sim 10^{-16} m[10]\right)$. Hence, the result we present in this work is of 
considerable theoretical interest and importance. Our derivation, though naive, gives an order of magnitude size of the minimal length that can be considered operational in nuclear systems. After all, minimal length can be considered to be of the same size as the Compton wavelength of the particles under consideration; above that it may also depend on the interaction strength. This is a matter which is under investigation and will be dealt with somewhere else, but for now, the estimation we did here is close to the Compton wavelength of deuteron $\left(\sim 10^{-16} \mathrm{~m}\right)$ times the square of nuclear interaction strength $(\sim$ $0.1)$. Therefore, we hope we will be able to construct a theoretical framework which will cast more light on minimal length applicable for atomic and nuclear systems at energy scale much below the Planck scale.

\section{Conclusion}

Quantum gravity, string theory etc conclusively indicate towards a minimal length which is a length below which no resolution of space is possible. This minimal length modifies ordinary Heisenberg uncertainty principle and gives rise to the generalized uncertainty principle (GUP). The framework of GUP is by now well established and many studies of the influence of this principle on ordinary quantum mechanics (see, e.g., [13-17]) and relativistic quantum mechanics (see, e.g., [18-22]) are now available in literature. GUP influences not only Planck scale physics, which is the main domain of the minimal length, but also much larger length scale. And this is due to the UV-IR mixing which is inherent in the GUP. Due to this matter of fact, the minimal length, usually in operation at Planck scale, may show up as a length determined by the nature of the quantum systems one deals with. Thus the upperbound on minimal length appears as $\sim 10^{-17} \mathrm{~m}$ from studies of hydrogen atom. In this present work, we modify the quantum mechanics of the deuteron problem using generalized uncertainty principle and work out the extra contribution to binding energy of deuteron from the generalized uncertainty principle. Comparing that with the experimental uncertainty of the binding energy of deuteron, we find out an upperbound on minimal length inferable from the binding energy of deuteron. The upperbound we found is close to the value found using the hydrogen atom binging energy. Hence, we think our work is very illuminating to the field of minimal length-quantum mechanics.

\section{References}

1. A. Kempf, J. Math. Phys. 35, 4483 (1994). https://doi.org/10.1063/1.530798

2. H. Hinrichsen and A. Kempf, J. Math. Phys. 37, 2121(1996). https://doi.org/10.1063/1.531501

3. A. Kempf, J. Phys. A: Math. Gen. 30, 2093 (1997). https://doi.org/10.1088/0305$\underline{4470 / 30 / 6 / 030}$

4. L. J. Garay, Int. J. Mod. Phys. 10, 145 (1995). https://doi.org/10.1142/S0217751X95000085

5. K. Konishi, G. Paffuti, and P. Provero, Phys. Lett. B 234, 276 (1990). https://doi.org/10.1016/0370-2693(90)91927-4

6. R. J. Szabo, Phys. Rep. 378, 207 (2003). https://doi.org/10.1016/S0370-1573(03)00059-0

7. A. Kempf, G. Mangano, and R. B. Mann, Phys. Rev. D 52, 1108 (1995). https://doi.org/10.1103/PhysRevD.52.1108

8. F. Brau, J. Phys. A: Math. Gen. 32, 7691 (1999). https://doi.org/10.1088/0305-4470/32/44/308 
9. F. Brau and F. Buisseret, Phys. Rev. D 74, 036002 (2006).

https://doi.org/10.1103/PhysRevD.74.036002

10. S. B. Faruque, M. A. Rahman, and M. Moniruzzaman, Res. Phys. 4, 52 (2014).

11. C. V. Leun and C. Alderliesten, Nucl. Phys. A 380, 261(1982). https://doi.org/10.1016/03759474(82)90105-1

12. J. L. Powell and B.Crasemann, Quantum Mechanics (Addison-Wesley Pub, USA, 1961).

13. L. N. Chang, D. Minic, N. Okamura, and T. Takeuchi, Phys. Rev. D 65, 125027 (2002). https://doi.org/10.1103/PhysRevD.65.125027

14. A. Camacho, Gen. Relativ. Gravit. 35, 1153 (2003). https://doi.org/10.1023/A:1024437522212

15. D. Bouaziz and M. Bawin, Phys. Rev. A 76, 032112 (2007). https://doi.org/10.1103/PhysRevA.76.032112

16. M. M. Stesko and V. M. Tkachuk, Phys. Rev. A 76, 012707 (2007). https://doi.org/10.1103/PhysRevA.76.012707

17. K. Nozari and P. Pedram, Europhys. Lett. 92, 50013 (2010). https://doi.org/10.1209/02955075/92/50013

18. C. Quesne and V. M. Tkachuk, J. Phys. A: Math. Gen. 38, 1747 (2005). https://doi.org/10.1088/0305-4470/38/8/011

19. S. Hossain and S. B. Faruque, Phys. Scr. 78, 035006 (2008). https://doi.org/10.1088/0031$\underline{8949 / 78 / 03 / 035006}$

20. M. Moniruzzaman and S. B. Faruque, Phys. Scr. 82, 035005 (2010). https://doi.org/10.1088/0031-8949/82/03/035005

21. M. Moniruzzaman and S. B. Faruque, Phys. Scr. 85, 035006 (2012). https://doi.org/10.1088/0031-8949/85/03/035006

22. K. Jahankohan, H. Hassanabadi, and S. Zarrinkamar, Mod. Phys. Lett. A 30, 1550173 (2015). https://doi.org/10.1142/S0217732315501734 